\title{
AC MAGNE'TIC MEASUREMENTS OF THE ALS BOOSTER DIPOLE ENGINEERING MODEL MAGNET *
}

\author{
M. I. Green, E. Hoyer, R. Keller, and D. H. Nelsen \\ Lawrence Berkeley Laboratory \\ University of California \\ Berkelev. CA 94720 USA
}

\section{$\underline{\text { Abstract }}$}

$10 \mathrm{~Hz}$ sine wave and $2 \mathrm{~Hz}$ sawtooth $\mathrm{AC}$ magnetic measurements of the curved ALS Booster Dipole Engineering Model Magnet have been accomplished. Long curved coils were utilized to measure the integral transfer function and uniformity. Point coils and a Hall Probe were used to measure magnetic induction and its uniformity. The data were logged and processed by a Tektronix 11401 digital oscilloscope. The dependence of the effective length on the field was determined from the ratio of the integral coil signals to the point coil signals. Quadrupole and sextupole harmonics were derived from the point and integral uniformity measurements

\section{Introduction}

The ALS is a third generation, $1-2 \mathrm{GeV}$ synchrotron radiation facility specifically designed to maximize the brightness of the radiation from wigglers and undulators 1 . This project includes a low-emittance electron storage ring optimized at $1.5 \mathrm{GeV}$, an injection system which includes a $50 \mathrm{MeV}$ linac and the $1.5 \mathrm{GeV}$ booster synchrotron, and a complement of insertion devices and photon beam lines. Twenty-four dipole magnets will provide the main guide field for the booster synchrotron. The synchrotron is intended to operate at $1 \mathrm{~Hz}$, but the magnets are designed for $10 \mathrm{~Hz}$ operation.

The booster synchrotron dipole magnet is of the split $\mathrm{H}$ type with hat pancake coils. 2 To minimize the stored energy and power requirements, the core is curved to follow the electron-beam trajectory. Magnet design parameters are briefly tabulated in a companion paper at this conference. ${ }^{3}$ An engineering model of this dipole magnet has been designed, fabricated, and qualified through magnetic measurements and is now in production.

We made a minimal set of AC magnetic measurements of the engineering model of the ALS Booster Dipole Magnet as part of the process of qualifying its design for production. Magnetic induction integrals over paths approximating electron-beam trajectories were measured with long curved coils connected to an electronic integrator. ${ }^{3}$ Magnetic induction was measured with point coils and an integrator and independently with a Hall-effect Gaussmeter. These quantities, and magnet current, were displayed on a commercial digital storage oscilloscope as parametric functions of time.

The displayed waveforms were stored, processed and redisplayed as representations of selected magnet parameters. A waveform representing the magnet's effective length was created by dividing the integral waveform by the magnetic induction waveform. Waveforms of the transfer functions were produced by dividing both the integral waveform and the magnetic induction waveform by the current waveform. Pairs of matched coils, connected in series opposition, provided differential measurements of field uniformity. Quadrupole and sextupole coefficients were derived from the uniformity data.

These magnet parameters were measured at 2 and $10 \mathrm{~Hz}$ frequencies. Together with measurements of the magnetic field at selected DC levels, the $A C$ measurements demonstrated that the magnet design meets specifications and qualified it for production.

\section{Magnetic Field Parameters for Qualification}

The objective of the magnetic measurement effort for the booster dipole engineering model was to qualify the magnet design for production. Verification of the 2-D magnetostatic design and evaluation of the higher order field terms (quadrupole, sextupole, etc) largely generated at the ends, were mostly done with DC measurements. ${ }^{4} \mathrm{AC}$ measurements were carried out largely to investigate magnet and vacuum chamber eddy current effects. The measurements performed are tabulated in Table 1.

Table 1. Booster Dipole Engineering Model Magnetic Measurements

1. DC Measurements at selected field levels

1.1 Central Vertical-Field

1.2 Central Vertical-Field Integral

1.3 Midplane Vertical-Field Integral Uniformity

2. AC Measurements as Parametric Functions of Time

2.1 Central Vertical-Field Uniformity with/without the Vacuum Chamber

\subsection{Central Vertical-Field Integral}

\subsection{Midplane Vertical-Field Integral Uniformity}

\section{AC Measurement Techniques and Instrumentation}

Figure 1 shows test equipment configurations used for most of the $\mathrm{AC}$ measurements. Instrumentation features are tabulated in Table 2. Calibration procedures provided $0.1 \%$ absolute accuracy of both the Hall probe and the coil sensitivities and enabled us to match the sensitivity of coil pairs to better than $0.02 \%$. In operation we minimize the differential signal when one coil is on the magnet centerline and the bucking coil is at an arbitrary, stationary reference position $\left(\mathrm{x}=\mathrm{x}_{\mathrm{O}}\right)$.

Figure 1 also describes the magnet coordinate system used for the AC measurements. The origin of the Cartesian coordinate system is defined as the centroid of the volume between the pole tips; $+x$ extends the radius of curvature vector from the origin; $+y$ is the upward normal to the lower pole; $s$ is the distance from the origin along the curve consisting of a $15^{\circ}$ circular arc (defined by the magnet bend radius, $4.0107 \mathrm{~m}$ ) extended at each end by straight lines.

The measurement technique employed a Hall-effect Gaussmeter and point coils connected to an electronic integrator to measure magnetic induction. Magnetic induction integrals were measured with line coils shaped to conform to the nominal beam trajectory and connected to an electronic integrator. Magnet current was measured by use of a current monitoring resistor (shunt) in series with the magnet and its power supply. Data acquisition was with a digital storage oscilloscope (DSO) where signals from the Gaussmeter, integrator, and shunt were recorded and stored as parametric functions of time $(t)$. The DSO allowed two signals to be acquired simultaneously.

\footnotetext{
* This work was supported by the Director, Office of Energy Research, Office of Basic Energy Sciences, Materials Sciences Division, of the U.S. D.O.E., under Contract No. DE-AC03-76SF00098.
} 


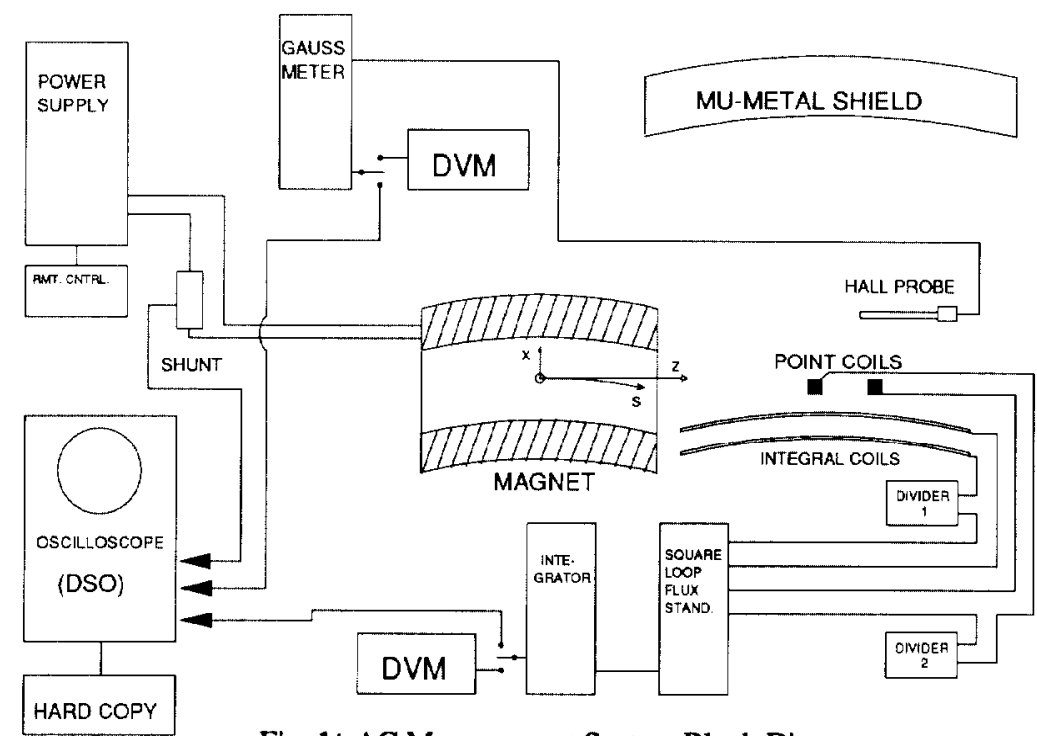

Fig. 1: AC Measurement System Block Diagram.

Table 2. Instrumentation and features

Digital Storage Oscilloscope

$500 \mathrm{MHz}$ bandwidths

Tektronix Model No. 11401

Multiple 10 Bit (1,024-point) waveform records

10-ps horizontal resolution

Full programmability through IEEE-488 or RS-232C (not

implemented)

Simplificd acquisition \& processing features available by "touchscreen" control

Electronic Integrator

LBL Model No 7

1-MicroVolt Second resolution (over several minute periods)

Point Coils \& Line Coils

LBL Designs

Absolute accuracy $+/-0.1 \%$

Relative accuracy $+1-0.02 \%$

Flux Standard

Absolute accuracy $+1-0.02 \%$

Relative accuracy $+1-0.01 \%$

Range: .01 - 1.0 Vs selectable in $1,2,5$ steps

Hall Effect Gaussmeter

Absolute accuracy $0.25 \%$ of full scale

$+/-0.1 \%$ with linearity correction and in field calibration and zero/offset procedures

For determining transfer functions and effective length, a single pair of signals was acquired, i.e. The integrator signal and the shun signal for determining the transfer function waveform, and two integrator outpul signals for determining the effective length function.

We developed an $\mathrm{AC}$ measurement technique whereby variations in effective length $\left(\Delta \mathrm{L}_{\mathrm{eff}}\right)$ are determined from differential measurements as follows:

$$
\Delta L_{\text {eff }}=\frac{\left\{\mathrm{B}_{\mathrm{y}}(0,0) \mathrm{ds}-\mathrm{B}_{\mathrm{y}}\left(\mathrm{x}_{0}, 0,0\right) \mathrm{L}_{\mathrm{eq}}\left(\mathrm{B}^{*}\right)\right\}(\mathrm{t})}{\left\{\mathrm{B}_{\mathrm{y}}(0,0,0)\right\}(t)}=\mathrm{L}_{\text {eff }}(\mathrm{t})-\mathrm{L}_{\mathrm{eq}}\left(\mathrm{B}^{*}\right)
$$

The numerator is the differential signal obtained by connecting the point coil in series opposition to the line coil. Leq (equivalent) $\left(B^{*}\right)$ is numerically equal to Leff $\left(t^{*}\right)$, the effective length evaluated at $t=t^{*}$, i.e., when $\left\{B_{y}(0,0,0)\right\}\left(t=t^{*}\right)=B^{*}$. Leq $\left(B^{*}\right)$ is realized by adjusting the point-coil divider such that its divided signal precisely cancels the line coil's signal at $t=t^{*}$. Dividing this differential signal waveform by the magnetic induction waveform yields the difference in effective length as shown by the equation. To compute $L_{\text {eff }}(t)$ the effective length $L_{e f f}\left(t^{*}\right)$, determined independently, is added to $\Delta L_{\text {eff. }}$ This technique overcomes the accuracy limitation in determining the effective length by the ratio of two parametric functions whose resolution is limited by the resolution of the oscilloscope.

For determining the field uniformity on the aperture midplane, a series of differential waveforms was acquired with a pair of matched coils electrically connected in series opposition. The acquired waveforms corresponded to the field at the $x$-position of one of the coils $\left(x=x_{i}\right)$ with respect to the field at an arbitrary, stationary, $x$ position of the second (bucking) coil $\left(x=x_{0}\right)$, i.e.,

$\left\{\Delta B\left(x_{i}\right)=B\left(x_{i}\right)-B\left(x_{0}\right)\right\}$ for the local magnetic induction uniformity and $\left\{\Delta \int B d s\left(x_{i}\right)=\int B d s\left(x_{i}\right)-\int B d s\left(x_{0}\right)\right\}$ for the integral uniformity. Each series started and ended with the first coil located on the aperture centerline $\left(x_{i}=0\right)$. The averaged waveforms at $x_{i}=$ $o$ were subtracted from each of the other waveforms. This process partially compensated for integrator drift between the first and last measurements, although this drift was negligible.

\section{Results}

\section{Effective Length}

The accuracy of the acquired data was limited by the $10 \mathrm{Bit}$ resolution of the oscilloscope to $0.1 \%$ of the maximum signal size or $1.25 \times 10^{-3} \mathrm{~T}$ which is about $3 \%$ of the minimum field. We were able to increase the resolution by expanding the waveform into a region of interest with 10 Bit resolution at low fields.

The DC and $2 \mathrm{~Hz}$ measured effective length data is graphed and described in a companion paper at this conference. 5

\section{Eield Uniformity}

Field uniformity measurements were made with point coils, both with and without vacuum chamber sections, and with integral coils in order to distinguish between eddy-current induced effects, pole shape effects, and magnet end field effects on the field quality. Within the vacuum chamber (inserted between the poles) measurements were made at only three $x$-positions due to space restrictions. Data taken at three positions allowed determination of quadrupole and sextupole coefficients as is illustrated in Figure 2. 


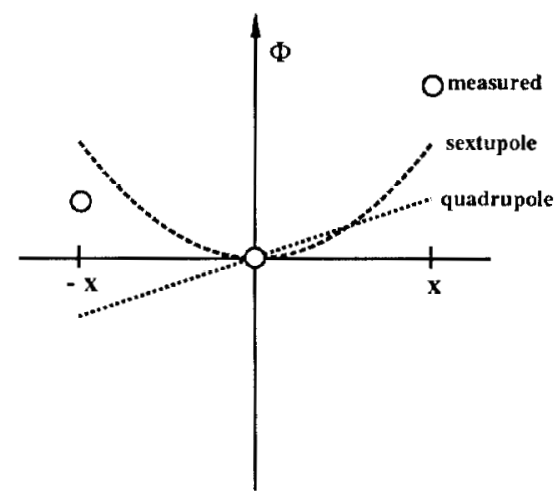

Fig. 2. Derivation of quadrupole and sextupole coefficients from magnet field data measured at three locations across the magnet.

Results obtained from $2 \mathrm{~Hz}$ and $10 \mathrm{~Hz}$ measurements are displayed in Figures. 3- 4. Data that represent point coil measurements have to be multiplied by the effective length, to be compared to the integral data. Only those results are displayed that appear not to be affected by the discussed data acquisition accuracy problems.

The integrated sextupole values, measured both at $D C$ and at 2 and $10 \mathrm{~Hz}$ without a vacuum chamber are plotted and described in a companion paper at this conference. 5

In Figure 3 the local sextupole strengths, measured near the magnet center without the vacuum chamber, are plotted for both 2 and $10 \mathrm{~Hz}$ excitations. The curves for 2.3 and $3.2 \mathrm{~cm}$ are essentially flat with negligible amplitude, showing slight saturation effects only at the highest field levels investigated. This means that the transverse pole contours, optimized with a 2-dimensional simulation code, fulfill the quality requirements outlined above.

Figure 4 shows local sextupole strengths measured near the magnet center - at $2 \mathrm{llz}$ with a $0.8-\mathrm{mm}$ thick stainless steel vacuum chamber and at $10 \mathrm{~Hz}$ with a $0.3 \mathrm{~mm}$ thick chamber. Strong eddy current effects are seen at the lower field levels as expected.

When the Booster Ring is operational, with yacuum chambers in place, the sextupole strength will be determined by three effects: eddy currents, end geometry, and saturation. The first and last of these are time-dependent, each one being significantly strong when the other one is negligible. Due to the opposite signs of eddy-curnent induced and geometrical sextupoles, the absolute value of the total sextupole strength for the entire excitation cycle is moderate.

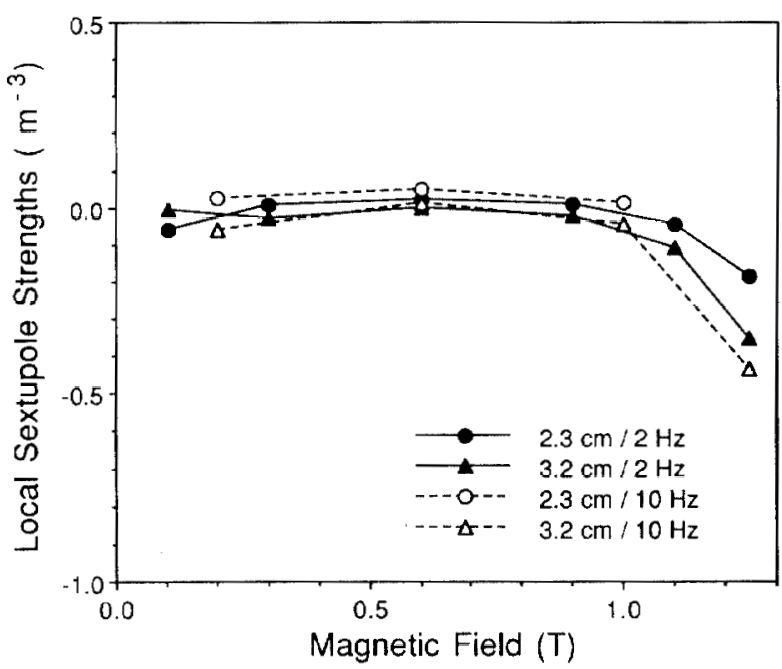

Fig. 3. Local sextupole values without vacuum chamber.

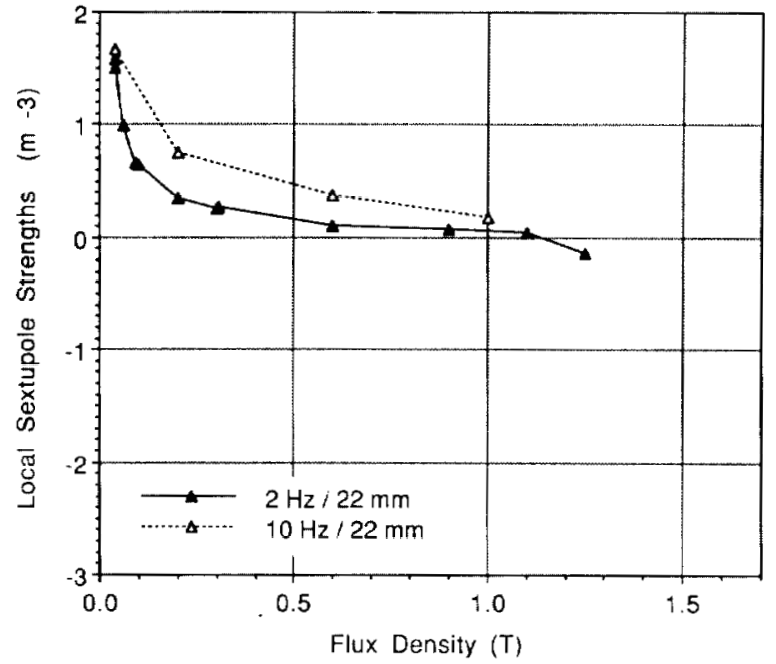

Fig. 4. Local sextupole values with vacuurn chamber installed. Note: The $2 \mathrm{~Hz}$ curve is composed from three different measurement series.

\section{Conclusion}

The AC magnetic qualities of the ALS Booster Dipole were measured at 2 and $10 \mathrm{~Hz}$ frequencies. Many measurements, taken over the entire excitation range, showed intolerably high errors at low field values due to the resolution of the data acquisition hardware used. However, by combining measurements that were taken over narrower ranges and in one case by employing a differential measurement technique, these errors were compensated for. The magnetic length and field uniformity values obtained demonstrate that the magnet design meets the specifications and qualify it for production.

\section{Acknowledgements}

The authors wish to thank Klaus Halbach, LBL, for the idea of using a storage oscilloscope; Mike Lapolla, Tektronix, for making the Tektronix 11401 digital oscilloscope available to LBL; John Cerino and his staff at SSRL for making the SPEAR injector prototype $10 \mathrm{~Hz}$ power supply available; and to various members of the AlS project team for their help. We especially thank Sharon Fujimura and David VanDyke for their assistance in preparing this paper and Yang Mo Koo for help in acquiring data.

\section{References}

[1] "1-2 GeV Synchrotron Radiation Source", Conceptual Design Report, LBL PUB-5172, July, 1986.

[2] E. Hoyer, "Light Source Booster Dipole Magnet Design Calculations", LBL Engineering Note M6673, Dec. 1987.

[3] M.I. Green, D. Nelson, S. Marks, B. Gee, W. Wong, and J. Meneghetti, "Design, Fabrication, and Calibration of Curved Integral Coils for Measuring Transfer Function, Uniformity, and Effective Length of LBL ALS Booster Dipole Magnets", Proceedings of this Conference.

[4] R. Keller, M.I. Green, E. Hoyer, Y.M. Koo, and D.H. Nelson, "Measured Properties of the ALS Booster Synchrotron Bending Magnet Engineering Model", Internal Report LSAP61, Lawrence Berkeley Laboratory, 1988.

[5] R. Keller, M.I. Green, E. Hoyer, Y.M. Koo, K. Luchini, S. Marks, J. Milburn, and D.H. Nelson, "Magnetic Properties of the ALS Booster Synchrotron Engineering Model Magnets", Proceedines of this Conference. 